\title{
SLNR-based Precoder Design in Multiuser Interference Channel with Channel Estimation Error
}

\author{
Bangwon Seo ${ }^{1^{*}}$ \\ ${ }^{1}$ Electrical, Electronics and Control Engineering, the Institute of IT Convergence Technology (IICT), \\ Kongju National University, Cheonan-si, Seobuk-gu, Chungnam, South Korea \\ [e-mail: seobw@kongju.ac.kr] \\ *Corresponding author: Bangwon Seo
}

Received August 29, 2018; revised December 28, 2018; revised June 14, 2019; accepted September 6, 2019; published January 31, 2020

\begin{abstract}
In this paper, we consider a precoder design problem for multiuser interference channel. Most of the conventional schemes for precoder design utilize a signal-to-interference-plus-noise ratio (SINR) as a cost function. However, since the SINR metric of a desired transmitter-receiver pair is a function of precoding vectors of all transmitters in the multiuser interference channel, an analytic closed-form solution is not available for the precoding vector of a desired transmitter that maximizes the SINR metric. To eliminate coupling between the precoding vectors of all transmitters and to find a closed-form solution for the precoding vector of the desired transmitter, we use a signal-to-leakage-plus-noise ratio (SLNR) instead as a cost function because the SLNR at a transmitter is a function of the precoding vector of the desired transmitter only. In addition, channel estimation errors for undesired links are considered when designing the precoding vector because they are inevitable in a multiuser interference channel. In this case, we propose a design scheme for the precoding vector that is robust to the channel estimation error. In the proposed scheme, the precoding vector is designed to maximize the worst-case SLNR. Through computer simulation, we show that the proposed scheme has better performance than the conventional scheme in terms of SLNR, SINR, and sum rate of all users.
\end{abstract}

Keywords: Channel estimation error, multiuser interference channel, precoder design, signal-to-leakage-plus noise ratio (SLNR). 


\section{Introduction}

In multiuser interference channel, there are multiple transmitters and multiple receivers, and each transmitter is intended to transmit information symbols to its intended receiver. Because a signal from a transmitter is received at all unwanted receivers as well as at a desired target receiver, the signal works as interference to the non-target receivers. This kind of interference is one of the major factors affecting performance degradation especially in multiuser interference channels [1]-[10].

If a transmitter is equipped with multiple transmit antennas, well-known multiple antenna transmission schemes can be applied at the transmitter to reduce interference in the multiuser interference channel. One promising multiple-antenna transmission scheme is a precoding scheme by which information symbols are multiplied by a precoding vector at a transmitter; then transmitted to a receiver [3]-[24]. In [11] and [12], the authors propose block diagonalization (BD)-based precoding schemes for multiuser interference channel where cochannel multiuser interference is perfectly eliminated at the cost of noise enhancement. However, these schemes impose a critical restriction that the number of transmit antennas must be larger than the number of the sum of receive antennas of all users. In [13] and [14], the authors propose precoding schemes based on the maximization of the received signal-to-interference-plus-noise ratio (SINR). These schemes do not cause noise enhancement problem and do not require any constraint on the number of transmit antennas. However, the SINR expression of any user is a function of precoding vectors of all users and all precoding vectors should be jointly designed. Due to the coupled property of the precoding vectors in the SINR-maximization-based design, it is impossible to find an analytical closedform solution of the SINR-based precoding vector design problem. The authors in [24] and [25] propose iteration methods to find an optimal precoding vector instead of a closed-form solution.

In order to decouple the joint optimization problems of all precoding vectors into multiple separate optimization problems of only target precoding vector and to find a closed-form solution for the target precoding vector, the authors in [26] propose a signal-to-leakage-plus-noise ratio (SLNR) metric-based precoding vector design scheme for the transmission of a single data stream in multiuser interference channel. Leakage at a transmitter refers to the interference caused by the signal intended for a target receiver to non-target receivers. Since the SLNR of a user is a function of the precoding vector of the user only, it is possible to find an analytical closed-form solution of the precoding vector that maximizes the SLNR metric. In [27], the authors extend the single stream SLNR based design scheme to multiple-stream transmission case. The precoding matrix for multiple streams of each user are obtained by the multiple-stream SLNR cost function and the generalized eigenvalue decomposition (GED) of the channel covariance matrix and the leakage channel-plus-noise covariance matrix of each user. However, in this scheme, the effective channel gain for each stream can be severely unbalanced and it causes a significant loss in the overall error performance of each user, especially when power control or adaptive modulation and coding cannot be applied. In [28], the authors present an SLNR-based precoding scheme for multiple-stream transmission case that reduces the margin between the effective SINRs of multiple data streams. By introducing a slight relaxation for pursuing SLNR maximization, they obtain a general form of simultaneous diagonalization of two covariance matrices linked to the user's channel and leakage-plus-noise. 
For multiuser interference channel, the SLNR metric for a transmitter is a function of channel vectors between the transmitter and all receivers including unwanted receivers. In the conventional SLNR-based precoder design schemes [26]-[28], the authors assume that the channel vectors for all links are perfectly known to the desired transmitter. Actually, the channel vector between the desired transmitter and its intended receiver can be easily estimated with good accuracy using reference signals. However, since it is very difficult to accurately estimate the channel vectors between the transmitter and its unwanted receivers, it is more reasonable to assume that there are channel estimation errors in the estimated channel vectors between a transmitter and its non-target receivers. Moreover, in general, the transmitter is not capable of knowing the exact statistics of the channel estimation error.

Contrary to the conventional schemes [26]-[28], in this paper we assume that the Frobenius norm of the channel estimation error matrix is bounded by some value and that each transmitter is capable of knowing only the boundary value instead of the exact statistics of the channel estimation error matrix. Then, we consider a robust precoder design problem for a multiuser interference channel when there are channel estimation errors in the links between a transmitter and its non-target receivers. To decouple a cost function for designing the precoding vector of a transmitter from the precoding vectors of other transmitters, we use an SLNR metric as a cost function because the SLNR of a transmitter is a function of the precoding vector of the desired transmitter only, and is independent of the precoding vectors of other transmitters. Considering the channel estimation error for the interference link, we propose a precoding vector design scheme that is robust to channel estimation errors and herein derive an optimal closed-form solution by maximizing the worst-case SLNR. Through computer simulations, we show that the proposed scheme has better performance than the conventional scheme in terms of SLNR, SINR, and the sum rate of all users.

The rest of the paper is organized as follows. In Section 2, the system model is described. In Section 3, a robust precoder design scheme for interference channel is proposed by considering channel estimation errors. The simulation results are given in Section 4. Finally, conclusions are presented in Section 5.

Notations: We use bold upper case letters to denote matrices, bold lower case letters to denote vectors. The notations $|\cdot|,\|\cdot\|$, and $\|\cdot\|_{F}$ denote the absolute, 2-norm, and Frobenius norm operations, respectively. Also, $(\cdot)^{H}, \operatorname{tr}(\cdot)$, and $E[\cdot]$ denote Hermitian transpose, trace, and expectation operations, respectively. The identity matrix of size $M \times M$ is denoted by $\mathbf{I}_{M}$. The notation max_geig $(\mathbf{A}, \mathbf{B})$ returns the normalized dominant generalized eigenvector of matrix pair $(\mathbf{A}, \mathbf{B})$.

\section{System Model}

We consider a multiuser interference channel. Fig. 1 shows the system model where there are $K$ transmitters and $J$ receivers. We assume that $K$ is equal to $J$ in multiuser interference channel. The transmitter $k$ transmits information symbols to the target receiver $k$ and its signal works as interference to the non-target receivers. In the figure, $\mathrm{TX} k$ and $\mathrm{RX} j$ with $k=1,2, \ldots, K, j=1,2, \ldots, J$ mean transmitter $k$ and receiver $j$, respectively. For simplicity, we assume that each transmitter is equipped with $M$ antennas and each receiver is equipped with a single antenna. One use case of this scenario is Internet of Things (IoT) communications where many IoT devices will be equipped with a single receive antenna each. 


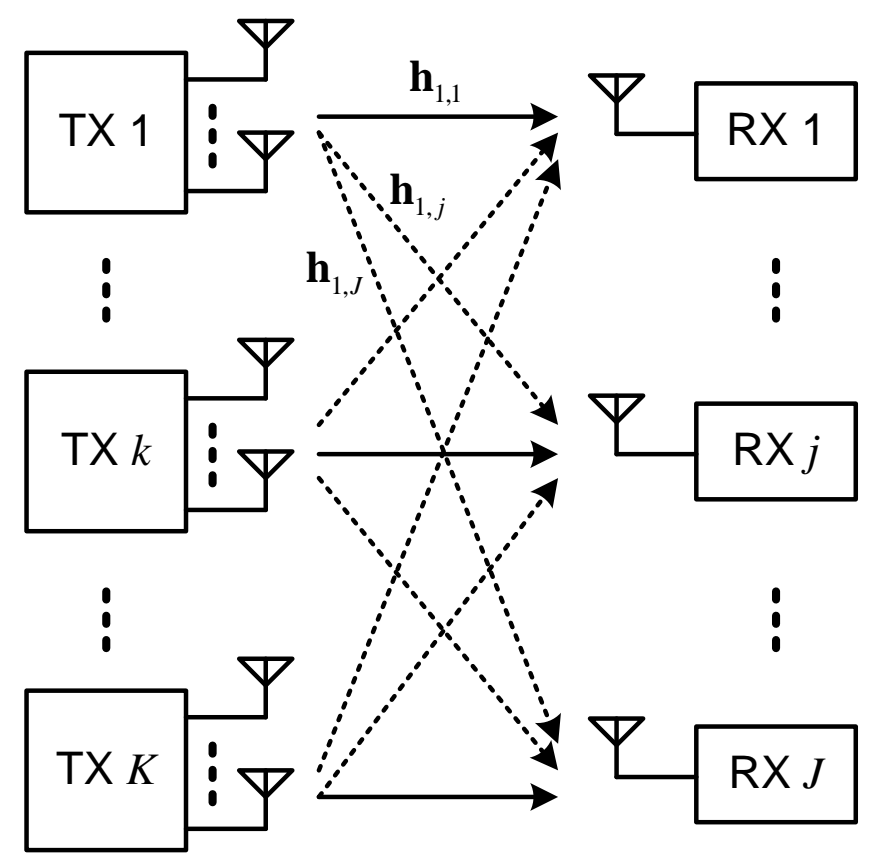

Fig. 1. Multiuser interference channel.

The channel vector for the link from TX $k$ to $\mathrm{RX} j$ is denoted by $\mathbf{h}_{k, j}$ of size $M \times 1$. In order to reduce inter-channel interference, TX $k$ applies a precoding vector $\mathbf{w}_{k}$ of size $M \times 1$ to its information symbol, $s_{k}$, with $E\left[s_{k}\right]=0$ and $E\left[\left|s_{k}\right|^{2}\right]=1$. Then, the received signal at $\mathrm{RX} j$ is represented by

$$
y_{j}=\sqrt{P_{j}} \mathbf{h}_{j, j}^{H} \mathbf{w}_{j} s_{j}+\sum_{k=1, k \neq j}^{K} \sqrt{P_{k}} \mathbf{h}_{k, j}^{H} \mathbf{w}_{k} s_{k}+z_{j}, j=1,2, \ldots, J
$$

where $P_{k}$ is the transmit power at TX $k$ and $z_{j}$ is an additive white Gaussian noise (AWGN) with zero mean and variance $\sigma_{z}^{2}$. We assume that $\mathbf{w}_{k}$ is normalized as $\left\|\mathbf{w}_{k}\right\|^{2}=\mathbf{w}_{k}^{H} \mathbf{w}_{k}=1$. On the right side of equation (1), the first term is the desired signal for $\operatorname{RX} j$, the second one represents inter-channel interference, and the third one is AWGN.

The SINR at $\mathrm{RX} \mathrm{j}$ is given by

$$
\begin{aligned}
\operatorname{SINR}_{j} & =\frac{E\left[\left\|\sqrt{P_{j}} \mathbf{h}_{j, j}^{H} \mathbf{w}_{j} s_{j}\right\|^{2}\right]}{E\left[\left\|\sum_{k=1, k \neq j}^{K} \sqrt{P_{k}} \mathbf{h}_{k, j}^{H} \mathbf{w}_{k} s_{k}+z_{j}\right\|^{2}\right]} \\
& =\frac{P_{j} \mathbf{w}_{j}^{H} \mathbf{h}_{j, j} \mathbf{h}_{j, j}^{H} \mathbf{w}_{j}}{\sum_{k=1, k \neq j}^{K} P_{k} \mathbf{w}_{k}^{H} \mathbf{h}_{k, j} \mathbf{h}_{k, j}^{H} \mathbf{w}_{k}+\sigma_{z}^{2}} .
\end{aligned}
$$


In most conventional schemes, the precoding vector $\mathbf{w}_{j}$ is designed to maximize $\operatorname{SINR}_{j}$. However, because SINR $_{j}$ is a function of all precoding vectors, $\mathbf{w}_{1}, \mathbf{w}_{2}, \ldots, \mathbf{w}_{K}$, all precoding vectors should be jointly designed by considering SINRs of all RXs, the $\operatorname{SINR}_{j}, j=1,2, \ldots, J$. Therefore, a closed-form solution for this joint optimization problem is not available and iteration schemes have been proposed to solve the joint optimization problem instead.

One way to isolate the optimization problems to find the precoding vectors is to use SLNR at TX $k$ as a cost function. Leakage represents the interference with non-target receivers caused by a signal intended for a target receiver. The leakage power from $\mathrm{TX} k$ to $\mathrm{RX} j$ for $k \neq j$ is expressed by $P_{k}\left|\mathbf{w}_{k}^{H} \mathbf{h}_{k, j}\right|^{2}$. The SLNR at TX $k$ is denoted by

$$
\begin{aligned}
\operatorname{SLNR}_{k} & =\frac{P_{k} \mathbf{w}_{k}^{H} \mathbf{h}_{k, k} \mathbf{h}_{k, k}^{H} \mathbf{w}_{k}}{\sum_{j=1, j \neq k}^{J} P_{k} \mathbf{w}_{k}^{H} \mathbf{h}_{k, j} \mathbf{h}_{k, j}^{H} \mathbf{w}_{k}+\sigma_{z}^{2}} \\
& =\frac{P_{k} \mathbf{w}_{k}^{H} \mathbf{h}_{k, k} \mathbf{h}_{k, k}^{H} \mathbf{w}_{k}}{\mathbf{w}_{k}^{H}\left(\sum_{j=1, j \neq k}^{J} P_{k} \mathbf{h}_{k, j} \mathbf{h}_{k, j}^{H}+\sigma_{z}^{2} \mathbf{I}_{M}\right) \mathbf{w}_{k}},
\end{aligned}
$$

where $\mathbf{I}_{M}$ is an identity matrix of size $M \times M$.

From equation (3), we can observe that $\mathrm{SLNR}_{k}$ is a function of $\mathbf{w}_{k}$ only and it is independent of $\mathbf{w}_{n}, n=1,2, \ldots, K, n \neq k$. The optimal precoding vector $\mathbf{w}_{k}^{\text {opt }}$ is obtained by solving the following optimization problem:

$$
\mathbf{w}_{k}^{\mathrm{opt}}=\arg \max _{\mathbf{w}_{k}} \mathrm{SLNR}_{k} .
$$

Therefore, $\mathbf{w}_{k}^{\text {opt }}$ is given by the generalized eigenvector as follows [26]:

$$
\mathbf{w}_{k}^{\mathrm{opt}}=\max \_ \text {geig }\left(\mathbf{h}_{k, k} \mathbf{h}_{k, k}^{H} \sum_{j=1, j \neq k}^{J} \mathbf{h}_{k, j} \mathbf{h}_{k, j}^{H}+\frac{\sigma_{z}^{2}}{P_{k}} \mathbf{I}_{M}\right),
$$

where max_geig(A,B) returns the normalized dominant generalized eigenvector of matrix pair $(\mathbf{A}, \mathbf{B})$.

\section{Proposed Robust Precoding Vector Design}

In this section, we propose a robust precoding vector design scheme in multiuser interference channel considering channel estimation error.

From equation (3), we can see that the channel vectors for the links between TX $k$ and all $\mathrm{RXs}$ are required to calculate the SLNR at TX $k$. In general, TX $k$ is able to know the channel vector for the desired link between TX $k$ and RX $k$ with a good accuracy using reference signals transmitted from TX $k$. However, it is not easy to accurately estimate the channel 
vector for the interference link between $\operatorname{TX~} k$ and $\operatorname{RX} j, j \neq k$. Hence, it is reasonable to assume that TX $k$ is able to know $\mathbf{h}_{k, k}$ perfectly, but there is a channel estimation error in the estimate of $\mathbf{h}_{k, j}, j=1,2, \ldots, J, j \neq k$.

In this section, by considering the channel estimation error, we propose a precoding vector design scheme that is robust to the channel estimation error. In order to consider channel estimation error for the interference link, we define the average SLNR at TX $k$ by

$$
\overline{\operatorname{SLNR}}_{k}=E\left[\mathrm{SLNR}_{k}\right]=\frac{P_{k} \mathbf{w}_{k}^{H} \mathbf{h}_{k, k} \mathbf{h}_{k, k}^{H} \mathbf{w}_{k}}{\mathbf{w}_{k}^{H}\left(\sum_{j=1, j \neq k}^{J} P_{k} E\left[\mathbf{h}_{k, j} \mathbf{h}_{k, j}^{H}\right]+\sigma_{z}^{2} \mathbf{I}_{M}\right) \mathbf{w}_{k}} .
$$

In order to consider the channel estimation error for the link between $\operatorname{TX} k$ and $\mathrm{RX} j$, we assume that the true channel vector $\mathbf{h}_{k, j}$ is represented by the sum of its estimate $\hat{\mathbf{h}}_{k, j}$ and the channel estimation error $\mathbf{e}_{k, j}$ as follows:

$$
\mathbf{h}_{k, j}=\hat{\mathbf{h}}_{k, j}+\mathbf{e}_{k, j},
$$

where $\mathbf{e}_{k, j}$ has zero mean and covariance matrix $\mathbf{Q}_{k, j}=E\left[\mathbf{e}_{k, j} \mathbf{e}_{k, j}^{H}\right]$. Moreover, we assume that the Frobenius norm of $\mathbf{Q}_{k, j}$ is bounded by $\varepsilon_{k, j}$ (i.e., $\left.\left\|\mathbf{Q}_{k, j}\right\|_{F}^{2}=\operatorname{tr}\left(\mathbf{Q}_{k, j} \mathbf{Q}_{k, j}^{H}\right) \leq \varepsilon_{k, j}^{2}\right)$ and TX $k$ is able to know only the bound value $\varepsilon_{k, j}, j=1,2, \ldots, J, j \neq k$, instead of $\mathbf{Q}_{k, j}$ itself.

Then, $E\left[\mathbf{h}_{k, j} \mathbf{h}_{k, j}^{H}\right]$ in the equation (6) can be rewritten as $E\left[\mathbf{h}_{k, j} \mathbf{h}_{k, j}^{H}\right]=\hat{\mathbf{h}}_{k, j} \hat{\mathbf{h}}_{k, j}^{H}+\mathbf{Q}_{k, j}$ and $\overline{\mathrm{SLNR}}_{k}$ is expressed as follows:

$$
\overline{\operatorname{SLNR}}_{k}=\frac{P_{k} \mathbf{w}_{k}^{H} \mathbf{h}_{k, k} \mathbf{h}_{k, k}^{H} \mathbf{w}_{k}}{\mathbf{w}_{k}^{H}\left(\sum_{j=1, j \neq k}^{J} P_{j}\left(\hat{\mathbf{h}}_{k, j} \hat{\mathbf{h}}_{k, j}^{H}+\mathbf{Q}_{k, j}\right)+\sigma_{z}^{2} \mathbf{I}_{M}\right) \mathbf{w}_{k}} .
$$

Because TX $k$ is able to know only the bound value of the Frobenius norm of $\mathbf{Q}_{k, j}$ for the interference link, we propose to maximize the worst-case SLNR in designing the precoding vector. The worst-case SLNR means the minimum value of SLNR for all $\mathbf{Q}_{k, j}, j=1,2, \ldots, J, j \neq k$, satisfying the bound condition $\left\|\mathbf{Q}_{k, j}\right\|_{F}^{2}=\operatorname{tr}\left(\mathbf{Q}_{k, j} \mathbf{Q}_{k, j}^{H}\right) \leq \varepsilon_{k, j}^{2}$. Hence, the worst-case SLNR is given by

$$
\overline{\operatorname{SLNR}}_{k, \text { min }}=\min _{\mathbf{Q}_{k, 1}, \mathbf{Q}_{k, 2}, \ldots, \mathbf{Q}_{k, k}} \overline{\operatorname{SLNR}}_{k} \text { subject to } \operatorname{tr}\left(\mathbf{Q}_{k, j} \mathbf{Q}_{k, j}^{H}\right) \leq \varepsilon_{k, j}^{2} \text { for } j=1,2, \ldots, J, j \neq k .
$$

In order to apply the Lagrange multiplier method, we modify equation (9) with an equivalent form as follows: 


$$
\begin{aligned}
& \overline{\operatorname{SLNR}}_{k, \min }= \min _{\mathbf{Q}_{k, 1}, \mathbf{Q}_{k, 2}, \ldots, \mathbf{Q}_{k, J}}-1 / \overline{\operatorname{SLNR}}_{k} \text { subject to } \operatorname{tr}\left(\mathbf{Q}_{k, j} \mathbf{Q}_{k, j}^{H}\right) \leq \varepsilon_{k, j}^{2} \text { for } j=1,2, \ldots, J, j \neq k \\
&=\min _{\mathbf{Q}_{k, 1}, \mathbf{Q}_{k, 2} \cdots, \mathbf{Q}_{k, J}}-\frac{\mathbf{w}_{k}^{H}\left(\sum_{j=1, j \neq k}^{J} P_{j}\left(\hat{\mathbf{h}}_{k, j} \hat{\mathbf{h}}_{k, j}^{H}+\mathbf{Q}_{k, j}\right)+\sigma_{z}^{2} \mathbf{I}_{N}\right) \mathbf{w}_{k}}{P_{k} \mathbf{w}_{k}^{H} \mathbf{h}_{k, k} \mathbf{h}_{k, k}^{H} \mathbf{w}_{k}} \\
& \text { subject to } \operatorname{tr}\left(\mathbf{Q}_{k, j} \mathbf{Q}_{k, j}^{H}\right) \leq \varepsilon_{k, j}^{2} \text { for } j=1,2, \ldots, J, j \neq k .
\end{aligned}
$$

Now, this optimization problem can be solved using the Lagrange multiplier method [29]. We define the Lagrange multiplier by

$$
\begin{gathered}
L\left(\mathbf{Q}_{k, 1}, \mathbf{Q}_{k, 2}, \ldots, \mathbf{Q}_{k, J}, \lambda_{1}, \ldots, \lambda_{J}\right)=-\frac{\mathbf{w}_{k}^{H}\left(\sum_{j=1, j \neq k}^{J} P_{j}\left(\hat{\mathbf{h}}_{k, j} \hat{\mathbf{h}}_{k, j}^{H}+\mathbf{Q}_{k, j}\right)+\sigma_{z}^{2} \mathbf{I}_{M}\right) \mathbf{w}_{k}}{P_{k} \mathbf{w}_{k}^{H} \mathbf{h}_{k, k} \mathbf{h}_{k, k}^{H} \mathbf{w}_{k}} \\
+\sum_{j=1, j \neq k}^{J} \lambda_{j} \operatorname{tr}\left(\mathbf{Q}_{k, j} \mathbf{Q}_{k, j}^{H}\right)-\varepsilon_{k, j}^{2} .
\end{gathered}
$$

Derivatives of $L\left(\mathbf{Q}_{k, 1}, \mathbf{Q}_{k, 2}, \ldots, \mathbf{Q}_{k, J}, \lambda_{1}, \ldots, \lambda_{J}\right)$ by $\mathbf{Q}_{k, j}$ and $\lambda_{j}$ are given by

$$
\begin{aligned}
& \frac{\partial L}{\partial \mathbf{Q}_{k, j}}=\frac{-\mathbf{w}_{k} \mathbf{w}_{k}^{H}}{P_{k} \mathbf{w}_{k}^{H} \mathbf{h}_{k, k} \mathbf{h}_{k, k}^{H} \mathbf{w}_{k}}+\lambda_{j} \mathbf{Q}_{k, j}=0, \\
& \frac{\partial L}{\partial \lambda_{j}}=\operatorname{tr}\left(\mathbf{Q}_{k, j}^{H} \mathbf{Q}_{k, j}\right)-\varepsilon_{k, j}^{2}=0 .
\end{aligned}
$$

From the equation (12), we obtain

$$
\mathbf{Q}_{k, j}^{\mathrm{opt}}=\frac{\mathbf{w}_{k} \mathbf{w}_{k}^{H}}{\lambda_{j} P_{k} \mathbf{w}_{k}^{H} \mathbf{h}_{k, k} \mathbf{h}_{k, k}^{H} \mathbf{w}_{k}} .
$$

By substituting (14) into (13), we obtain

$$
\lambda_{j}^{\mathrm{opt}}=\frac{\left\|\mathbf{w}_{k}\right\|^{2}}{\varepsilon_{k, j} P_{k}\left\|\mathbf{w}_{k}^{H} \mathbf{h}_{k, k}\right\|^{2}} .
$$

From equation (14) and (15), we obtain

$$
\mathbf{Q}_{k, j}^{\mathrm{opt}}=\varepsilon_{k, j} \mathbf{w}_{k} \mathbf{w}_{k}^{H}
$$

By substituting (16) into (8), the worst-case SLNR for TX $k, \overline{\operatorname{SLNR}}_{k \text {,min }}$, is given by 


$$
\overline{\operatorname{SLNR}}_{k, \text { min }}=\frac{P_{k} \mathbf{w}_{k}^{H} \mathbf{h}_{k, k} \mathbf{h}_{k, k}^{H} \mathbf{w}_{k}}{\mathbf{w}_{k}^{H}\left(P_{k} \sum_{j=1, j \neq k}^{J} \hat{\mathbf{h}}_{k, j} \hat{\mathbf{h}}_{k, j}^{H}+\left((J-1) P_{k} \varepsilon_{k, j}+\sigma_{z}^{2}\right) \mathbf{I}_{M}\right) \mathbf{w}_{k}} .
$$

Now, we design the optimal vector $\mathbf{w}_{k}$ that maximizes $\overline{\operatorname{SLNR}}_{k, \min }$. The worst-case optimization problem for $\mathbf{w}_{k}$ is given by

$$
\overline{\operatorname{SLNR}}_{k, \text { min }}^{\max }=\max _{\mathbf{w}_{k}} \overline{\operatorname{SLNR}}_{k, \min } .
$$

The optimal vector $\mathbf{w}_{k}$ is obtained by the generalized eigenvector as follows:

$$
\mathbf{w}_{k}^{\text {opt }}=\max \text { _geig }\left(\mathbf{h}_{k, k} \mathbf{h}_{k, k}^{H}, \sum_{j=1, j \neq k}^{J} \hat{\mathbf{h}}_{k, j} \hat{\mathbf{h}}_{k, j}^{H}+\left((J-1) \varepsilon_{k, j}+\frac{\sigma_{z}^{2}}{P_{k}}\right) \mathbf{I}_{M}\right) .
$$

By applying the Shannon capacity formula, the sum rate of all TXs is given by

$$
\Gamma_{\text {sum }}=\sum_{j=1}^{J} \log _{2}\left(1+\operatorname{SINR}_{j}\right) \text {, }
$$

where $\operatorname{SINR}_{j}$ is obtained by inserting $\mathbf{w}_{j}^{\text {opt }}$ into (2).

\section{Simulation Results}

We performed a computer simulation to compare the performance of the proposed precoder with a conventional scheme [26] that does not consider channel estimation errors. The elements of the channel vectors are generated with independent and identically distributed (i.i.d.) complex Gaussian variables with zero mean and unit variance. For simplicity, we assume that the bound values for the Frobenius norm of the channel estimation error covariance matrices are the same for all transmission links (i.e., $\varepsilon_{k, 1}=\varepsilon_{k, 2}=\cdots=\varepsilon_{k, J}$ ). Moreover, we assume that the number of TXs is equal to that of RXs (that is, $K=J=3$ ) and the number of transmit antennas of each TX is $M=4$.

In Fig. 2, the SLNR performance of the proposed scheme is compared with the conventional scheme [26] for various bound value of the Frobenius norm of $\mathbf{Q}_{k, j}$ (i.e., $\left.\varepsilon_{k, j}=0.1,0.05,0.01\right)$. From the figure, we can observe that the SLNR performances of the proposed scheme and the conventional scheme get worse as the bound value $\varepsilon_{k, j}$ increases. However, the proposed scheme has better SLNR performance than the conventional scheme for all $\varepsilon_{k, j}$ values. The performance gap between the proposed scheme and the conventional scheme becomes larger as $\varepsilon_{k, j}$ increases. When the channel estimation error is negligible (such as $\varepsilon_{k, j}=0.01$ ), the proposed scheme has almost the same performance as the conventional scheme. 


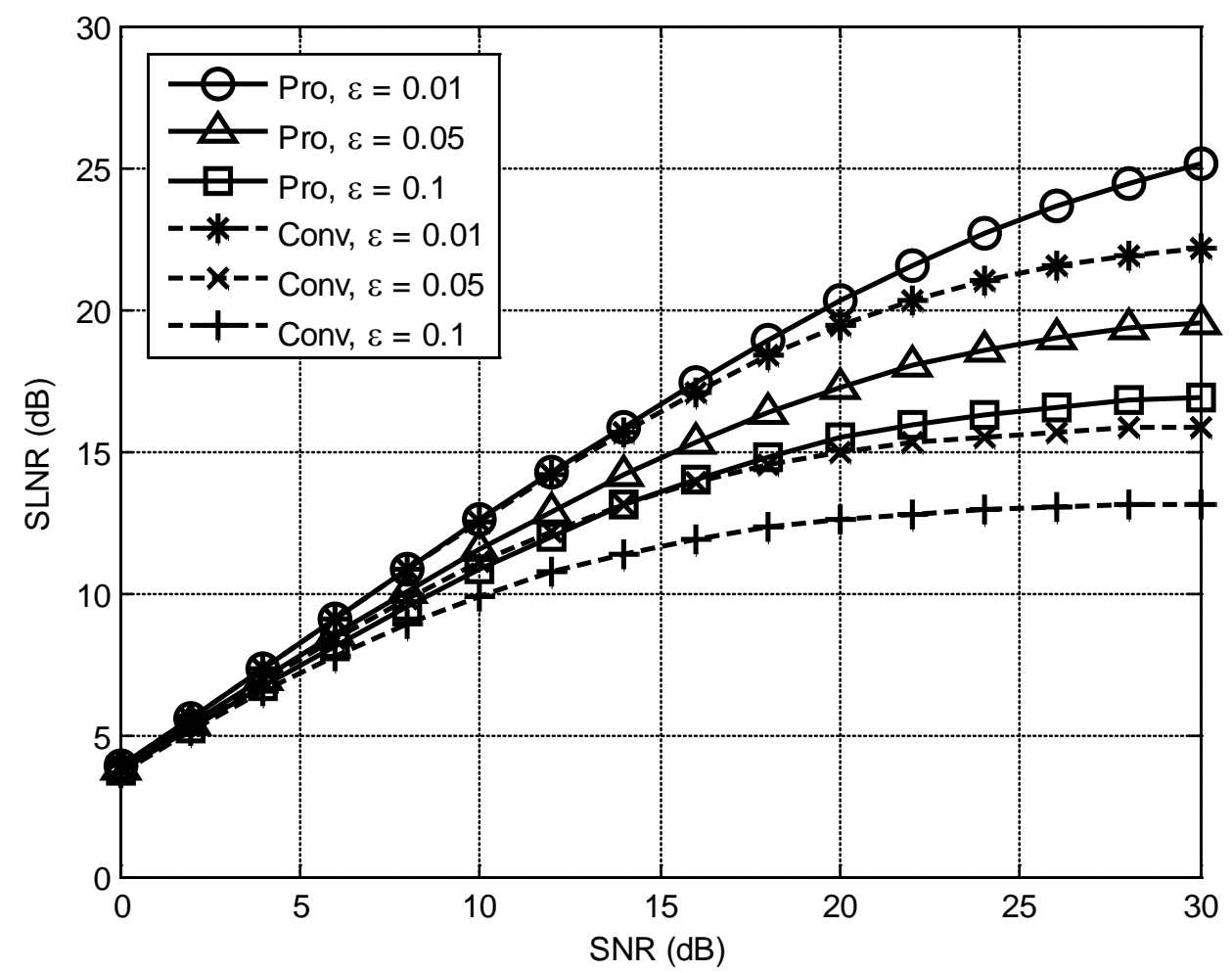

Fig. 2. SLNR performance comparison for different channel estimation error when $K=J=3$ and $M=4$.

Fig. 3 compares the SINR performance of the proposed scheme with that of the conventional scheme [26] when the number of transmitter and receiver pair is $K=3$ and the number of transmit antennas is $M=4$. From the figure, we can see that the proposed scheme has better SINR performance than the conventional scheme for all $\varepsilon_{k j}$. We can observe that the performance gap between the two schemes is higher when the error bound value $\varepsilon_{k j}$ is larger. This is because the conventional scheme [26] assumes that there is no channel estimation error in the interference channels between the transmitter and its unwanted receivers, but the channel estimation error becomes larger as the error bound value gets larger. On the other hand, the proposed scheme was designed to be robust to the channel estimation error by considering the estimation error bound. Also, we can observe that the performance gap between the proposed scheme and the conventional scheme becomes larger as the received SNR increases. The reason for this is that the channel estimation error of the interference channel is a more dominant factor than the background noise for the performance degradation in high SNR region. 


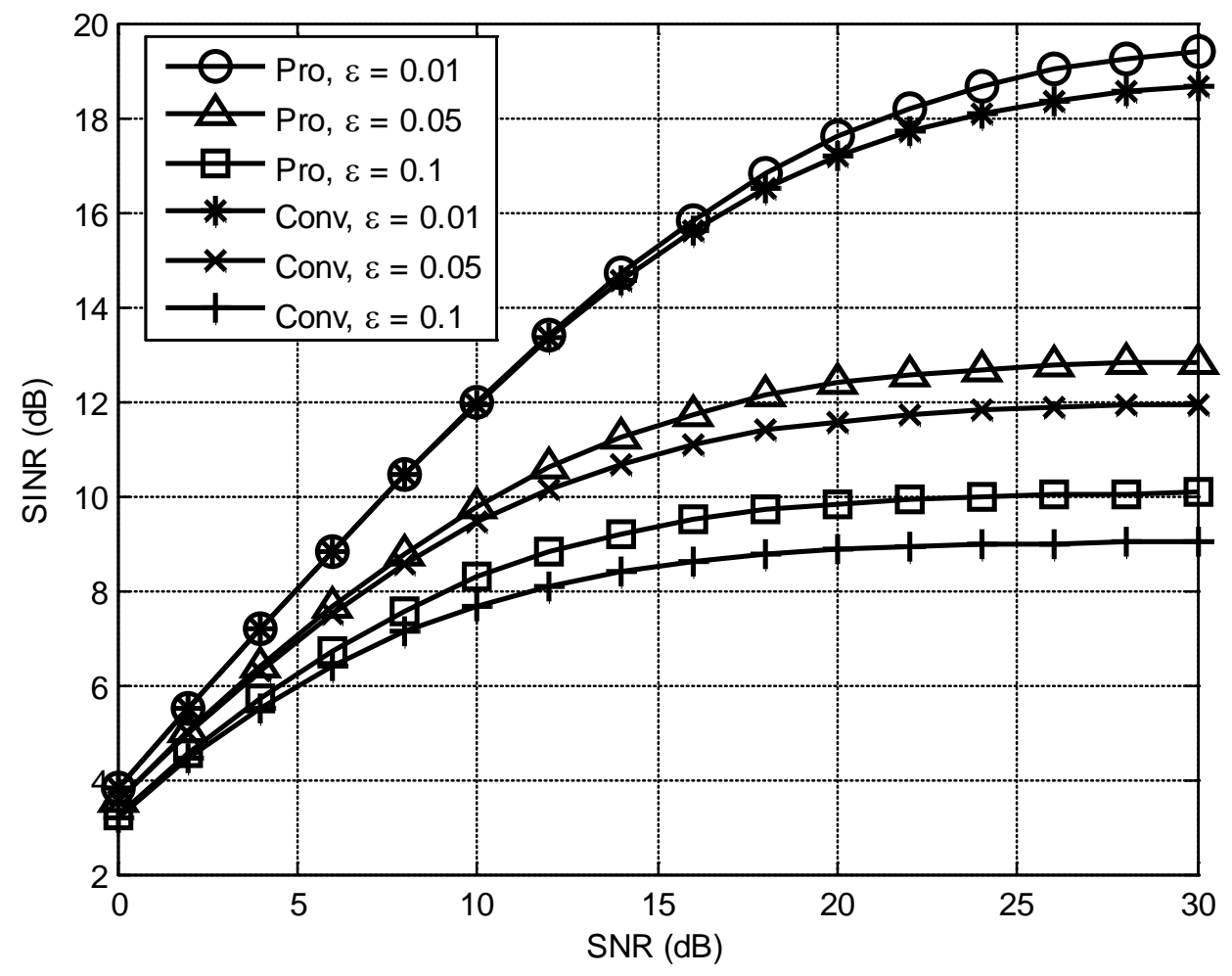

Fig. 3. SINR performance comparison for different channel estimation error when $K=J=3$ and $M=4$.

Fig. 4 shows the sum rate of all TX-RX links for the proposed scheme and the conventional scheme when the number of transmitter and receiver pair is $K=J=3$ and the number of transmit antennas is $M=4$. From the figure, we can observe that the sum rate of both proposed scheme a conventional schemes get worse as the bound value $\varepsilon_{k j}$ increases. Also, we can see that the proposed scheme has higher sum rate than the conventional scheme.

\section{Conclusions}

In this paper, we treated a precoder design for the interference channel by considering the channel estimation error for the interference link. We proposed a precoder design scheme that is robust to channel estimation errors by maximizing the worst-case SLNR metric. Through the simulation results, we showed that the proposed scheme has better performance than the conventional scheme in terms of SLNR, SINR, and sum rate of all users. 


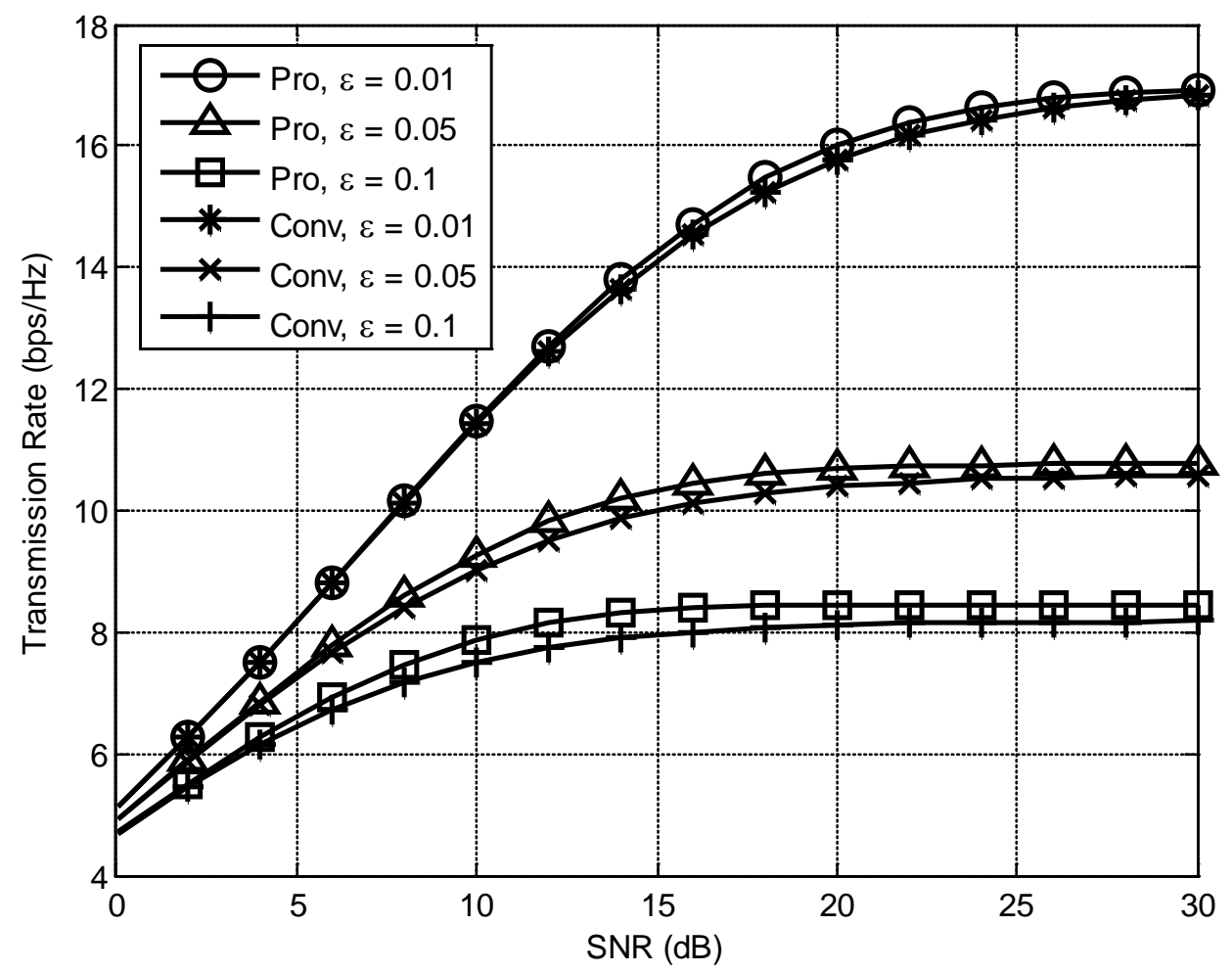

Fig. 4. Comparison of the sum rate for the different channel estimation error when $K=J=3$ and $M=4$.

\section{Acknowledgement}

This research was supported by Basic Science Research Program through the National Research Foundation of Korea(NRF) funded by the Ministry of Education (2016R1D1A3B03935210).

\section{References}

[1] A. S. Motahari and A. K. Khandani, "Capacity for the Gaussian interference channel," IEEE Trans. Inform. Theory, vol. 55, no. 2, pp. 620-643, Feb. 2009. Article (CrossRef Link).

[2] V. R. Cadambe and S. A. Jafar, "Interference alignment and degrees of freedom of the K-user interference channel," IEEE Trans. Inform. Theory, vol. 54, no. 8, pp. 3425-3441, Aug. 2008. Article (CrossRef Link).

[3] W. Zhong and Y. Xu, "Interference channel distributed energy efficient spectrum sharing strategy selection with limited feedback in MIMO interference channels," in Proc. of IEEE Global Telecomm. Conf. (GLOBECOM), pp. 1-5, Dec. 2010. Article (CrossRef Link).

[4] V. Sreekanth Annapureddy and V. V. Veeravalli, "Sum capacity of MIMO interference channels in the low interference regime,” IEEE Trans. Inform. Theory, vol. 57, no. 5, pp. 2565-2581, May 2011. Article (CrossRef Link).

[5] S. Akhalaghi and M. Baghani, "On the average achievable rate of block fading decentralized interference channel,” IEEE Comm. Lett., vol. 15, no. 9, pp. 992-994, Sept. 2011.

Article (CrossRef Link). 
[6] L. Zhou and W. Yu, "Gaussian Z-interference channel with a relay link: Achievability region and asymptotic sum capacity,” IEEE Trans. Inform. Theory, vol. 58, no. 4, pp. 2413-2426, Apr. 2012. Article (CrossRef Link).

[7] Y. Zhu and D. Guo, "The degrees of freedom of isotropic MIMO interference channels without state information at the transmitters," IEEE Trans. Inform. Theory, vol. 58, no.1, pp. 341-352, Jan. 2012. Article (CrossRef Link).

[8] H. Chang, S.-Y. Chung, and S. Kim, "Interference channel with a causal relay under strong and very strong interference,” IEEE Trans. Inform. Theory, vol. 60, no. 2, pp. 859-865, Feb. 2014. Article (CrossRef Link).

[9] K. Mohanty and M. K. Varanasi, "Degrees of freedom of the MIMO Z-interference channel with delayed CSIT,” IEEE Comm. Lett., vol. 19, no. 12, pp. 2282-2285, Dec. 2015. Article (CrossRef Link).

[10] E. Ebrahimzadeh, K. Moshksar, and A. K. Khandani, "Signaling over two-user parallel Gaussian interference channels: Outage analysis,” IEEE Trans. Inform. Theory, vol. 62, no. 5, pp. 2573-2591, May 2016. Article (CrossRef Link).

[11] Q. H. Spencer, A. L. Swindlehurst, and M. Haardt, "Zero-forcing methods for downlink spatial multiplexing in multi-user MIMO channels,” IEEE Trans. Signal Process., vol. 52, pp. 461-471, Feb. 2004. Article (CrossRef Link).

[12] T. Yoo and A. Goldsmith, "Optimality of zero-forcing beamforming with multiuser diversity," in Proc. of IEEE Inter. Conf. Commun. (ICC), Seoul, Korea, May 2005. Article (CrossRef Link).

[13] K. Wong, R Cheng, K. B. Letaeif, and R. D. Murch, "Adaptive antennas at the mobile and base station in an OFM/TDMA system,” IEEE Trans. Commun., vol. 49, pp. 195-206, Jan. 2001. Article (CrossRef Link).

[14] M. Schubert and H. Boche, "Solution of the multiuser downlink beamforming problem with individual SINR constraints,” IEEE Trans. Veh. Technol., vol. 53, no. 1, pp. 18-28, Jan. 2004. Article (CrossRef Link).

[15] H. Wang, L. Li, L. Song, and X. Gao, “A linear precoding scheme for downlink multiuser MIMO precoding systems,” IEEE Comm. Lett., vol. 15, no. 6, pp. 653-655, Jun. 2011. Article (CrossRef Link).

[16] L. Zhang, Z. Luo, S.-H. Leung, Y. Zhu, "Simplified precoder design for MIMO systems with receive correlation,” IEEE Signal Process., vol. 19, no. 5, pp. 263-266, May 2012. Article (CrossRef Link).

[17] P. Patcharamaneepakorn, S. Armour, and A. Doufexi, "On the equivalence between SLNR and MMSE precoding schemes with single-antenna receivers,” IEEE Comm. Lett., vol. 16, no. 7, pp. 1034-1037, Jul. 2012. Article (CrossRef Link).

[18] Y. Wu, M. Wang, C. Xiao, Z. Ding, and X. Gao, "Linear precoding for MIMO broadcast channels with finite-alphabet constraints,” IEEE Trans. Wireless Commun., vol. 11, no. 8, pp. 2906-2920, Aug. 2012. Article (CrossRef Link).

[19] X. Xia, S. Fang, G. Wu, and S. Li, "Joint User Pairing and Precoding in MU-MIMO Broadcast Channel with Limited Feedback,” IEEE Comm. Lett., vol. 14, no. 11, pp. 1032-1034, Nov. 2010. Article (CrossRef Link).

[20] V. Raghavan, S. Subramanian, J. Cezanne, A. Sampath, O. H. Koymen, and J. Li, "Single-user versus multi-user precoding for millimeter wave MIMO systems,” IEEE J. Sel. Areas Commun., vol. 35, no. 6, pp. 1387-1401, Jun. 2017. Article (CrossRef Link).

[21] Y. Liu and L. Dai, "Improving secrecy via extension to regularized channel inversion precoding," IEEE Comm. Lett., vol. 22, no. 5, pp. 1030-1033, May 2018. Article (CrossRef Link).

[22] H. Dai, Y. Huang, Y. Xu, C. Li, B. Wang, and L. Yang, "Energy-Efficient Resource Allocation for Energy Harvesting-Based Device-to-Device Communication,” IEEE Trans. Veh. Technol., vol. 68, no. 1, pp. 509-524, Jan. 2019. Article (CrossRef Link).

[23] C. Li, P. Liu, C. Zou, F. Sun, J. M. Cioffi, and L. Yang, "Spectral-efficient cellular communications with coexistent one- and two-hop transmissions,” IEEE Trans. Veh. Technol., vol. 65, no. 8, pp. 6765-6772, Aug. 2016. Article (CrossRef Link). 
[24] C. Li, C. He, L. Jiang, and F. Liu, "Robust beamforming design for max-min SINR in MIMO interference channel,” IEEE Commun. Lett., vol. 20, no. 4, pp. 724-727, Apr. 2016. Article (CrossRef Link).

[25] Y.-F. Liu, Y.-H. Dai, and Z.-Q, Luo, "Max-min fairness linear transceiver design problem for a multi-user MIMO interference channel,” IEEE Trans. Signal Process., vol. 61, no. 9, pp. 2413-2423, May 2013. Article (CrossRef Link).

[26] M. Sadek, A. Tarighat, and A. H. Sayed, "A leakage-based precoding scheme for downlink multi-user MIMO channels,” IEEE Trans. Wireless Commun., vol. 6, no. 5, pp. 1711-1721, May 2007. Article (CrossRef Link).

[27] M. Sadek and S. Aissa, "Leakage based precoding for multi-user MIMO-OFDM systems,” IEEE Trans. Wirel. Commun., vol. 10, no. 8, pp. 2428-2433, Aug. 2011. Article (CrossRef Link).

[28] P Cheng, M. Tao, and W, Zhang, “A new SLNR-based linear precoding for downlink multi-user multi-stream MIMO systems,” IEEE Commun. Lett., vol. 14, no. 11, pp.1008-1010, Nov. 2010. Article (CrossRef Link).

[29] S. Boyd and L. Vandenberghe, Convex Optimization, Cambridge University Press, Los Angeles, 2004. Article (CrossRef Link).

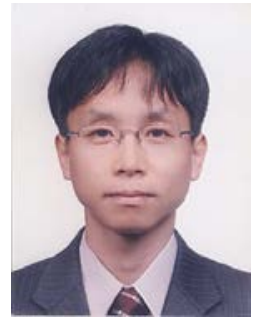

Bangwon Seo received the B.S., M.S., and Ph.D degrees in Electrical Engineering from the Korea Advanced Institute of Science and Technology (KAIST), Daejeon, South Korea, in 1997, 1999, and 2010, respectively. From November 2004 to February 2013, he was with the Electronics Telecommunications Research Institute (ETRI), Daejeon, Korea. In March 2013, he joined the Division of Electrical, Electronic and Control Engineering, Kongju National University, South Korea, where he is currently an Associate Professor. His research interests include massive MIMO, hybrid transceiver, device-to-device (D2D) direct communications, MIMO systems, and OFDM systems. 